\title{
Switching Signal Design for Exponential Stability of Uncertain Discrete-Time Switched Time-Delay Systems
}

\author{
Zheng-Fan Liu, Chen-Xiao Cai, and Yun Zou \\ School of Automation, Nanjing University of Science and Technology, Nanjing 210094, China \\ Correspondence should be addressed to Chen-Xiao Cai; ccx5281@vip.163.com
}

Received 4 June 2013; Accepted 9 August 2013

Academic Editor: Weihai Zhang

Copyright (c) 2013 Zheng-Fan Liu et al. This is an open access article distributed under the Creative Commons Attribution License, which permits unrestricted use, distribution, and reproduction in any medium, provided the original work is properly cited.

\begin{abstract}
The switching signal design for exponential stability with $H_{\infty}$ performance of uncertain switched linear discrete-time systems with interval time-varying delay is considered. Systems with norm-bounded parameter uncertainties are considered. By taking a new Lyapunov-Krasovskii (LK) function, sufficient conditions for the existence of a class of stabilizing switching laws are derived in terms of linear matrix inequalities (LMIs) to guarantee the considered switched time-delay system to be exponentially stable. The resulting stability criteria are of fewer matrix variables and are less conservative than some existing ones. In addition, numerical examples are illustrated to show the main improvement.
\end{abstract}

\section{Introduction}

Switched system is represented as the family of subsystems with switching rule which is concerned with various environmental factors and different controllers. During the last decades, there has been increasing interest in the stability analysis and control design for the switched systems (see, e.g., [1-6]). The design of switching signal is one of the three basic problems in stability analysis and the design of switched systems $[2,3]$. Switching signal included timedriven switching and state-driven switching. In the first case, time-driven switching depends on time, and many effective methods have been developed such as dwell time method [ 7 , $8]$, average dwell time method $[9,10]$, and mode-dependent average dwell time method $[11,12]$. In the case of state-driven switching, a switching action takes place when the system state hits a switching surface, and the study of the stability is based on a number of methods, including piecewise Lyapunov function [13] and convex combination [14].

On the other hand, time delay is one of the instability sources for dynamical systems and is a common phenomenon in many industrial and engineering systems. During the last two decades, much attention has been paid to the problem of stability analysis and controller synthesis for time-delay systems (see, e.g., [15-18]). A switched system with time-delay individual subsystems is called a switched time-delay system [19-28]. Switched time-delay systems have various applications in practical engineering systems, such as power systems and power electronics [1,21]. Many important results on the dynamical behavior have been reported for switched time-delay system [8-12].

Recently, increasing attention has been devoted to the problem of delay-dependent stability of switched delay systems [22-26]. In [23-25], a switching signal design technique is proposed to guarantee the asymptotic stability of switched systems with interval time-varying delay. Based on a discrete LK functional, in [26] a switching rule for the asymptotic stability and stabilization for a class of discretetime switched systems with interval time-varying delays is designed via linear matrix inequalities. However, the term $\sum_{s=k+1-d(k)}^{k-d_{m}} x^{T}(s) Q x(s)=\sum_{s=k+1-d_{M}}^{k-d_{m}} x^{T}(s) Q x(s)-$ $\sum_{s=k+1-d_{M}}^{k-d(k)} x^{T}(s) Q x(s)$ is estimated as $\sum_{s=k+1-d_{M}}^{k-d_{m}} x^{T}(s) Q x(s)$ for any $0<d_{m} \leq d(k) \leq d_{M}, Q=Q^{T}>0$, which may lead to considerable conservativeness. Following the work, free weighting matrix and additional nonnegative inequality approaches have been used to improve the conservativeness for the obtained results in [27]. However, many free weighting matrices were introduced, which made the stability result complicated. $H_{\infty}$ concept was proposed to reduce the effect of the disturbance input on the regulated output to 
within a prescribed level. In [29], $H_{\infty}$ controls were proposed for uncertain discrete switched systems under arbitrary switching signal. In [30], a switching signal design for $H_{\infty}$ performance of uncertain discrete switched systems with interval delay and linear fractional perturbations is considered. Nevertheless, the criteria still leave some room for improvement in accuracy as well as complexity due to the method used and offer motivation for further investigation.

Motivated by the above literatures, in this paper, by using an improved discrete LK function combined with LMIs technique, a new switching signal design approach is developed to guarantee the $H_{\infty}$ performance for uncertain discrete switched systems with interval time-varying delay to be exponentially stable. Numerical examples are given to show the effectiveness of the proposed method which can be easily solved by using MATLAB LMI control toolbox.

The remaining part of the paper is organized as follows. In Section 2, some preliminaries are introduced. In Section 3, the main results for uncertain switched linear discrete-time systems with interval time-varying delay are presented to be exponential stability. In Section 4, some numerical examples are given to illustrate the effectiveness and the merit of the proposed method. The last section concludes the work.

Notations. We use standard notations throughout the paper. $\lambda_{\text {min }}(M)\left(\lambda_{\max }(M)\right)$ stands for the minimal (maximum) eigenvalue of $M . M^{T}$ is the transpose of the matrix $M$. The relation $M>N(M<N)$ means that the matrix $M-N$ is positive (negative) definite. $\|x\|$ denotes the Euclidian-norm of the vector $x \in R^{n}$. $R^{n}$ represents the $n$-dimensional real Euclidean space. $R^{n \times m}$ is the set of all real $n \times m$ matrices. $\operatorname{diag}\{\cdots\}$ stands for a block-diagonal matrix. In symmetric block matrices or long matrix expressions, we use an asterisk “*” to represent a term that is induced by symmetry. I denotes the identity matrix.

\section{Problem Description and Preliminaries}

Consider the following uncertain linear discrete-time switched time-delay system:

$$
\begin{aligned}
x(k+1)= & {\left[A_{\sigma}+\Delta A_{\sigma}(k)\right] x(k) } \\
& +\left[A_{d \sigma}+\Delta A_{d \sigma}(k)\right] x(k-d(k)) \\
& +\left[B_{\sigma}+\Delta B_{\sigma}(k)\right] w(k), \\
y(k)=[ & \left.C_{\sigma}+\Delta C_{\sigma}(k)\right] x(k) \\
+ & {\left[C_{d \sigma}+\Delta C_{d \sigma}(k)\right] x(k-d(k)) } \\
& +\left[D_{\sigma}+\Delta D_{\sigma}(k)\right] w(k), \\
x(l)= & \phi(l), \quad l=k_{0}-d_{M}, \ldots, k_{0},
\end{aligned}
$$

where $x(k) \in R^{n}$ denotes the system state vector. $y(k) \in R^{m}$ is the measured output. $w(k) \in R^{p}$ is the disturbance input which belongs to $l_{2}[0, \infty) . \phi(l) \in R^{n}$ is a vector-valued initial function. The switching signal $\sigma: Z \rightarrow \mho=\{1,2, \ldots, N\}$ is a piecewise constant function. $\sigma=i$ means that the $i$ th subsystem is activated. $N$ is the number of subsystems of the switched system. The system matrices $A_{i}, A_{d i}, B_{i}, C_{i}, C_{d i}$, and $D_{i}$ are a set of known real matrices with appropriate dimensions. $\Delta A_{i}(k), \Delta A_{d i}(k), \Delta B_{i}(k), \Delta C_{i}(k), \Delta C_{d i}(k)$, and $\Delta D_{i}(k)$ are real-valued unknown matrices representing time-varying parameter uncertainties, and are assumed to be of the form

$$
\begin{aligned}
& {\left[\begin{array}{ccc}
\Delta A_{i}(k) & \Delta A_{d i}(k) & \Delta B_{i}(k) \\
\Delta C_{i}(k) & \Delta C_{d i}(k) & \Delta D_{i}(k)
\end{array}\right]=\left[\begin{array}{l}
M_{1 i} \\
M_{2 i}
\end{array}\right]} \\
& \Delta_{1 i}(k)\left[\begin{array}{lll}
N_{1 i} & N_{2 i} & N_{3 i}
\end{array}\right],
\end{aligned}
$$

where $M_{1 i}, M_{2 i}, N_{1 i}, N_{2 i}$, and $N_{3 i}$ are known real constant matrices and $\Delta_{1 i}(k): N \rightarrow R^{l_{1} \times l_{2}}$ is unknown time-varying matrix function satisfying

$$
\Delta_{1 i}^{T}(k) \Delta_{1 i}(k) \leq I
$$

The parameter uncertainties $\Delta A_{i}(k), \Delta A_{d i}(k), \Delta B_{i}(k)$, $\Delta C_{i}(k), \Delta C_{d i}(k)$, and $\Delta D_{i}(k)$ are said to be admissible if both (2) and (3) hold.

For given finite positive integer $d_{m}$ and $d_{M}$, time-varying delay $d(k)$ satisfying

$$
0<d_{m} \leq d(k) \leq d_{M}, \quad \forall k \in N^{+} .
$$

Now we present the following definitions and lemmas that are useful in deriving the principal contribution of this paper.

Definition 1 (see [20]). The system (1) is said to be exponentially stable if there exist a switching function $\sigma(\cdot)$ and positive number $c$ such that any solution $x(k, \phi)$ of the system satisfies

$$
\|x(k)\| \leq c \lambda^{k-k_{0}}\|\phi\|_{s}, \quad \forall k \geq k_{0}
$$

for any initial conditions $\left(k_{0}, \phi\right) \in R^{+} \times C^{n} . c>0$ is the decay coefficient, $0<\lambda \leq 1$ is the decay rate, and $\|\phi\|_{s}=\sup \{\|\phi(l)\|$, $\left.l=k_{0}-d_{M}, k_{0}-d_{M}+1, \ldots, k_{0}\right\}$.

Definition 2 (see [26]). The system of matrices $\left\{L_{i}\right\} \quad(i \in \mho)$ is said to be strictly complete if, for every $x \in R^{n} \backslash\{0\}$, there is $i \in \mho$ such that $x^{T} L_{i} x<0$.

It is easy to see that the system of matrices $\left\{L_{i}\right\}(i \in \mho)$ is strictly complete if and only if $\bigcup_{i=1}^{N} \Omega_{i}=R^{n} \backslash\{0\}$, where $\Omega_{i}=$ $\left\{x \in R^{n}: x^{T} L_{i} x<0\right\}(i \in \mho)$.

Lemma 3 (see [31]). The system of matrices $\left\{L_{i}\right\}(i \in \mho)$ is strictly complete if there exist $\alpha_{i} \geq 0, \sum_{i=1}^{N} \alpha_{i}=1$ such that $\sum_{i=1}^{N} \alpha_{i} L_{i}<0$. If $N=2$; then the above condition is also necessary for the strict completeness.

Lemma 4 (see [17]). For any matrix $R=R^{T}>0$ integers $a \leq b$, if vector function $\xi(k):\{-b,-b+1, \ldots,-a\} \rightarrow R^{n}$, then

$$
(a-b) \sum_{s=k-b}^{k-a-1} z^{T}(s) R z(s) \leq \xi^{T}(k)\left[\begin{array}{cc}
-R & R \\
* & -R
\end{array}\right] \xi(k),
$$


where

$$
\begin{gathered}
z(k)=x(k+1)-x(k), \\
\xi^{T}(k)=\left[\begin{array}{ll}
x^{T}(k-a) & x^{T}(k-b)
\end{array}\right] .
\end{gathered}
$$

Lemma 5 (see [32], Schur's complement). Let $M, P$, and $Q$ be given matrices such that $Q>0$. Then

$$
\left[\begin{array}{cc}
P & M \\
* & -Q
\end{array}\right]<0 \Longleftrightarrow P+M Q^{-1} M^{T}<0 .
$$

The objective of this paper is to design a reasonable switching rule for discrete-time switched system (1) with time-varying delay satisfying (4) to guarantee that the system while be exponentially stable.

\section{Main Result}

In this section, we will divide the whole state space $R^{n}$ into $N$ subregions and then define a particular quadratic function in each sub region. Via an appropriate designed switching rule, the particular quadratic function in each sub region will decrease along the system trajectory with the corresponding subsystem. Consequently, the whole switched system remains exponentially stable.

Firstly, we will introduce the switching regions and the corresponding switching law. Given $P>0$ and $U>0$, define the domains by

$$
\Omega_{i}\left(P, U, A_{i}\right)=\left\{x(k) \in R^{n}: x^{T}(k) Y_{i} x(k)<0\right\},
$$

where $Y_{i}=A_{i}^{T} P A_{i}-U, i \in \mho$. that

From the similar proof of $[26,27]$, it can be easily obtained

$$
\bigcup_{i=1}^{N} \Omega_{i}=R^{n} \backslash\{0\} .
$$

Construct the following switching region:

$$
\begin{aligned}
& \bar{\Omega}_{1}=\Omega_{1}, \quad \bar{\Omega}_{2}=\Omega_{2} \backslash \bar{\Omega}_{1}, \quad \bar{\Omega}_{3}=\Omega_{3} \backslash \bar{\Omega}_{1} \backslash \bar{\Omega}_{2}, \ldots, \\
& \bar{\Omega}_{N}=\Omega_{N} \backslash \bar{\Omega}_{1} \backslash \ldots \backslash \bar{\Omega}_{N-1} .
\end{aligned}
$$

We can obtain $\bigcup_{i=1}^{N} \bar{\Omega}_{i}=R^{n} \backslash\{0\}$ and $\bar{\Omega}_{i} \cap \bar{\Omega}_{j}=\phi$, for all $i \neq j$, where $\phi$ is an empty set.

After dividing the whole state space $R^{n}$ into $N$ sub regions, we construct the switching signal as follows:

$$
\sigma(x(k))=i, \quad \forall x(k) \in \bar{\Omega}_{i}(i \in \mho) .
$$

In order to discuss robust stability of system (1), first, we consider the following nominal system without parametric uncertainties:

$$
\begin{gathered}
x(k+1)=A_{i} x(k)+A_{d i} x(k-d(k))+B_{i} w(k), \\
y(k)=C_{i} x(k)+C_{d i} x(k-d(k))+D_{i} w(k) .
\end{gathered}
$$

The following theorem gives a sufficient condition for the existence of an admissible reasonable switching rule for system (13) with disturbance input $w(k)=0$ to be exponentially stable.
Theorem 6. For some constants $\gamma \in(0,1]$ and $0 \leq \alpha_{i} \leq 1, i \in$ $\mho, \sum_{i=1}^{N} \alpha_{i}=1$, if there exist positive definite symmetric matrices $P, U, Q_{j}, R_{j}, j=1,2,3$, such that the following LMIs hold:

$$
\Xi=\left[\begin{array}{ccccc}
\phi_{11} & \phi_{12} & \phi_{13} & 0 & \phi_{15} \\
* & \phi_{22} & \phi_{23} & \phi_{24} & \phi_{25} \\
* & * & \phi_{33} & 0 & 0 \\
* & * & * & \phi_{44} & 0 \\
* & * & * & * & -W
\end{array}\right]<0,
$$

then the system (13) with time-varying delay satisfying (4) and $w(k)=0$ is globally exponentially stable with convergence rate $\lambda=\sqrt{\gamma}$ by the switching signal designed by (12).

$$
\text { Here }
$$

$$
\begin{aligned}
\phi_{11}= & U-\gamma P+Q_{1}+Q_{3}-\frac{\gamma^{d_{m}}}{d_{m}}\left(R_{1}+R_{3}\right) \\
\phi_{12}= & A_{i}^{T} P B_{i}, \quad \phi_{13}=\frac{\gamma^{d_{m}}}{d_{m}}\left(R_{1}+R_{3}\right), \\
\phi_{15}= & \left(A_{i}-I\right)^{T} W^{T}, \\
\phi_{22}= & B_{i}^{T} P B_{i}-\gamma^{d_{M}} Q_{3}-\frac{\gamma^{d_{M}}}{d_{M}-d_{m}}\left(2 R_{2}+R_{3}\right), \\
\phi_{23}= & \frac{\gamma^{d_{M}}}{d_{M}-d_{m}}\left(R_{2}+R_{3}\right), \\
\phi_{24}= & \frac{\gamma^{d_{M}}}{d_{M}-d_{m}} R_{2}, \\
\phi_{25}= & B_{i}^{T} W^{T}, \\
\phi_{33}= & \gamma^{d_{m}}\left(Q_{2}-Q_{1}\right)-\frac{\gamma^{d_{m}}}{d_{m}}\left(R_{1}+R_{3}\right) \\
& -\frac{\gamma^{d_{M}}}{d_{M}-d_{m}}\left(R_{2}+R_{3}\right), \\
\phi_{44}= & -\gamma^{d_{M}} Q_{2}-\frac{\gamma^{d_{M}}}{d_{M}-d_{m}} R_{2}, \\
W= & \left(d_{M}-d_{m}\right) R_{2}+d_{m} R_{1}+d_{M} R_{3} .
\end{aligned}
$$

Proof. Consider the following LK function for system (1):

$$
V(k)=V_{1}(k)+V_{2}(k)+V_{3}(k) .
$$


Here

$$
\begin{aligned}
V_{1}(k)= & x^{T}(k) P x(k), \\
V_{2}(k)= & \sum_{s=k-d_{m}}^{k-1} \gamma^{k-1-s} x^{T}(s) Q_{1} x(s) \\
& +\sum_{s=k-d_{M}}^{k-d_{m}-1} \gamma^{k-1-s} x^{T}(s) Q_{2} x(s) \\
& +\sum_{s=k-d(k)}^{k-1} \gamma^{k-1-s} x^{T}(s) Q_{3} x(s), \\
V_{3}(k)= & \sum_{\theta=-d_{m}}^{-1} \sum_{s=k+\theta}^{k-1} \gamma^{k-1-s} z^{T}(s) R_{1} z(s) \\
& +\sum_{\theta=-d_{M}} \sum_{s=k+\theta}^{k-1} \gamma^{k-1-s} z^{T}(s) R_{2} z(s) \\
& +\sum_{\theta=-d}^{-1} \sum_{s=k+\theta}^{k-1} \gamma^{k-1-s} z^{T}(s) R_{3} z(s),
\end{aligned}
$$

where $P, Q_{j}, R_{j}, j=1,2,3$ are positive definite symmetric matrices and $\gamma \in(0,1]$.

Now, we will show the decay estimation of $V(k)$ in (17) along the state trajectory of system (1). To this end, define

$$
V(k+1)-\gamma V(k)=\sum_{j=1}^{3} \widetilde{\Delta} V_{j}(k)
$$

Here

$$
\begin{aligned}
\tilde{\Delta} V_{1}(k)= & x^{T}(k)\left(A_{i}^{T} P A_{i}-\gamma P\right) x(k) \\
& +2 x^{T}(k) A_{i}^{T} P B_{i} x(k-d(k)) \\
& +x^{T}(k-d(k)) B_{i}^{T} P B_{i} x(k-d(k)), \\
\widetilde{\Delta} V_{2}(k)= & x^{T}(k)\left(Q_{1}+Q_{3}\right) x(k) \\
& -\gamma^{d_{m}} x^{T}\left(k-d_{m}\right)\left(Q_{1}-Q_{2}\right) x\left(k-d_{m}\right) \\
& -\gamma^{\tau(k)} x^{T}(k-d(k)) Q_{3} x(k-d(k)) \\
& -\gamma^{d_{M}} x^{T}\left(k-d_{M}\right) Q_{2} x\left(k-d_{M}\right) \\
\leq & x^{T}(k)\left(Q_{1}+Q_{3}\right) x(k) \\
& -\gamma^{d_{M}} x^{T}(k-d(k)) Q_{3} x(k-d(k))
\end{aligned}
$$

$$
\begin{aligned}
& -\gamma^{d_{m}} x^{T}\left(k-d_{m}\right)\left(Q_{1}-Q_{2}\right) x\left(k-d_{m}\right) \\
& -\gamma^{d_{M}} x^{T}\left(k-d_{M}\right) Q_{2} x\left(k-d_{M}\right)
\end{aligned}
$$

$$
\begin{aligned}
\tilde{\Delta} V_{3}(k)= & z^{T}(k)\left(\left(d_{M}-d_{m}\right) R_{2}+d_{m} R_{1}+d(k) R_{3}\right) z(k) \\
& -\sum_{s=k-d_{m}}^{k-1} \gamma^{k-s} z^{T}(s) R_{1} z(s) \\
& -\sum_{s=k-d_{M}}^{k-d_{m}-1}\left\{\gamma^{k-s} z^{T}(s) R_{2} z(s)\right\} \\
& -\sum_{s=k-d(k)}^{k-1} \gamma^{k-s} z^{T}(s) R_{3} z(s),
\end{aligned}
$$

while

$$
\begin{array}{r}
-\sum_{s=k-d(k)}^{k-1} \gamma^{k-s} z^{T}(s) R_{3} z(s) \\
=-\sum_{s=k-d_{m}}^{k-1}\left\{\gamma^{k-s} z^{T}(s) R_{3} z(s)\right\} \\
-\sum_{s=k-d(k)}^{k-d_{m}-1} \gamma^{k-s} z^{T}(s) R_{3} z(s), \\
\sum_{s-d_{m}-1} \gamma^{k-s} z^{T}(s) R_{2} z(s) \\
=-\sum_{s=k-d(k)}^{k-d_{m}-1}\left\{\gamma^{k-s} z^{T}(s) R_{2} z(s)\right\} \\
-\sum_{s=k-d_{M}}^{k-d(k)-1} \gamma^{k-s} z^{T}(s) R_{2} z(s) .
\end{array}
$$

So (24) could be

$$
\begin{aligned}
\tilde{\Delta} V_{3}(k) \leq & z^{T}(k)\left(\left(d_{M}-d_{m}\right) R_{2}+d_{m} R_{1}+d_{M} R_{3}\right) z(k) \\
& -\sum_{s=k-d_{m}}^{k-1} \gamma^{k-s} z^{T}(s)\left(R_{1}+R_{3}\right) z(s) \\
& -\sum_{s=k-d(k)}^{k-d_{m}-1} \gamma^{k-s} z^{T}(s)\left(R_{2}+R_{3}\right) z(s) \\
& -\sum_{s=k-d_{M}}^{k-d(k)-1} \gamma^{k-s} z^{T}(s) R_{2} z(s)
\end{aligned}
$$


From Lemma 4, we have

$$
\begin{aligned}
& -\sum_{s=k-d_{m}}^{k-1} \gamma^{k-s} z^{T}(s)\left(R_{1}+R_{3}\right) z(s) \\
& \quad \leq \frac{\gamma^{d_{m}}}{d_{m}} \xi_{1}^{T}(k)\left[\begin{array}{cc}
-R_{1}-R_{3} & R_{1}+R_{3} \\
* & -R_{1}-R_{3}
\end{array}\right] \xi_{1}(k), \\
& -\sum_{s=k-d(k)}^{k-d_{m}-1} \gamma^{k-s} z^{T}(s)\left(R_{2}+R_{3}\right) z(s) \\
& \quad \leq \frac{\gamma^{d_{M}}}{d_{M}-d_{m}} \xi_{2}^{T}(k)\left[\begin{array}{cc}
-R_{2}-R_{3} & R_{2}+R_{3} \\
* & -R_{2}-R_{3}
\end{array}\right] \xi_{2}(k), \\
& -\sum_{k-d(k)-1} \gamma^{k-s} z^{T}(s) R_{2} z(s) \\
& s=k-d_{M} \\
& \leq \frac{\gamma^{d_{M}}}{d_{M}-d_{m}} \xi_{3}^{T}(k)\left[\begin{array}{cc}
-R_{2} & R_{2} \\
* & -R_{2}
\end{array}\right] \xi_{3}(k),
\end{aligned}
$$

where

$$
\begin{gathered}
\xi_{1}^{T}(k)=\left[\begin{array}{ll}
x^{T}(k) & x^{T}\left(k-d_{m}\right)
\end{array}\right], \\
\xi_{2}^{T}(k)=\left[\begin{array}{ll}
x^{T}\left(k-d_{m}\right) & x^{T}(k-d(k))
\end{array}\right], \\
\xi_{3}^{T}(k)=\left[\begin{array}{ll}
x^{T}(k-d(k)) & x^{T}\left(k-d_{M}\right)
\end{array}\right] .
\end{gathered}
$$

Combining (21)-(27), it yields

$$
\begin{aligned}
V(k+1) & -\gamma V(k) \\
\leq & \xi^{T}(k) \\
& \times\left[\begin{array}{cccc}
\phi_{11}+A_{i}^{T} W A_{i} & \phi_{12}+A_{i}^{T} W A_{i} & \phi_{13} & 0 \\
* & \phi_{22}+B_{i}^{T} W B_{i} & \phi_{23} & \phi_{24} \\
* & * & \phi_{33} & 0 \\
* & * & * & \phi_{44}
\end{array}\right] \xi(k) \\
& +x^{T}(k) Y_{i} x(k),
\end{aligned}
$$

where

$$
\xi^{T}(k)=\left[\begin{array}{llll}
x^{T}(k) & x^{T}(k-d(k)) & x^{T}\left(k-d_{m}\right) & x^{T}\left(k-d_{M}\right)
\end{array}\right] .
$$

By Lemma 3 and condition (15), we know that the system of matrices $Y_{i}=A_{i}^{T} P A_{i}-U, i \in \mho$ is strictly complete, and the sets $\Omega_{i}$ and $\bar{\Omega}_{i}$ by (5) and (6) are well defined such that

$$
\begin{gathered}
\bigcup_{i=1}^{N} \Omega_{i}=R^{n} \backslash\{0\}, \quad \bigcup_{i=1}^{N} \bar{\Omega}_{i}=R^{n} \backslash\{0\}, \\
\bar{\Omega}_{i} \cap \bar{\Omega}_{j}=\Phi, \quad i \neq j .
\end{gathered}
$$

Therefore, for any $x(k) \in R^{n}, k>0$, there always exists an $i \in\{1,2, \ldots, N\}$ such that $x(k) \in \bar{\Omega}_{i}$. Choosing the switching rule (12), by Schur's complement of [32] with condition (14), leads to

$$
V(k+1)-\gamma V(k) \leq 0 \Longleftrightarrow V(k) \leq \gamma^{k} V(0) .
$$

By the system LK function (17), there always exist two positive constants $c_{1}, c_{2}$ such that

$$
c_{1}\|x(k)\|^{2} \leq V(k), \quad V(0) \leq c_{2}\|x(0)\|_{s}^{2} .
$$

Here

$$
\begin{gathered}
c_{1}=\lambda_{\min }(P) \\
c_{2}=\lambda_{\max }(P)+\sum_{j=1}^{3}\left(\lambda_{\max }\left(Q_{j}\right)+\lambda_{\max }\left(R_{j}\right)\right) .
\end{gathered}
$$

From (32) and (33), one obtains

$$
\|x(k)\| \leq \sqrt{\frac{c_{2}}{c_{1}}} \gamma^{k / 2}\|x(0)\|_{s} .
$$

By Definition 1, we know that the system (1) is exponentially stable with decay rate $\lambda=\sqrt{\gamma}$. This completes the proof.

In order to determine the exponentially stability with $H_{\infty}$ performance $\kappa$ of system (1), we need to introduce the following definition.

Definition 7 (see [30]). Consider system (1) with the switching signal in (12) and the following conditions.

(i) With $w(k)=0$, the system (1) is exponentially stable with convergence rate $0<\gamma<1$.

(ii) With zero initial conditions, the signals $w(k)$ and $y(k)$ are bounded by

$\sum_{k=0}^{\infty} \gamma^{-2 k} y^{T}(k) y(k) \leq \kappa^{2} \sum_{k=0}^{\infty} \gamma^{-2 k} w^{T}(k) w(k)$,

for all $w \in L_{2}(\gamma, 0, \infty), w \neq 0$ for constants $\kappa>0$ and $0<\gamma<1$. In the above conditions, the system (1) is exponentially stabilizable with $H_{\infty}$ performance $\kappa$ and convergence rate $\gamma$ by switching signal in (12).

Theorem 8. For some constants $\gamma \in(0,1]$ and $0 \leq \alpha_{i} \leq 1$, $\sum_{i=1}^{N} \alpha_{i}=1(i \in \mho)$, if there exist positive definite symmetric 
matrices $P, U, Q_{j}, R_{j}(j=1,2,3)$ such that (15) and the following LMIs hold:

$$
\left[\begin{array}{ccccccc}
\phi_{11} & \phi_{12} & \phi_{13} & 0 & \tilde{\phi}_{15} & C_{i}^{T} & \phi_{15} \\
* & \phi_{22} & \phi_{23} & \phi_{24} & \tilde{\phi}_{25} & C_{d i}^{T} & \phi_{25} \\
* & * & \phi_{33} & 0 & 0 & 0 & 0 \\
* & * & * & \phi_{44} & 0 & 0 & 0 \\
* & * & * & * & \tilde{\phi}_{55} & D_{i}^{T} & B_{i}^{T} W^{T} \\
* & * & * & * & * & -I & 0 \\
* & * & * & * & * & * & -W
\end{array}\right]<0,
$$

then the system (13) with time-varying delay satisfying (4) is globally exponentially stable with convergence rate $\lambda=\sqrt{\gamma}$ and $H_{\infty}$ performance $\kappa$ by the switching signal designed by (12).
Here

$$
\begin{gathered}
\widetilde{\phi}_{15}=A_{i}^{T} P B_{i}, \\
\widetilde{\phi}_{25}=A_{d i}^{T} P B_{i}, \\
\widetilde{\phi}_{55}=B_{i}^{T} P B_{i}-\kappa^{2} .
\end{gathered}
$$

Proof. The proof is similar to that of Theorem 6. From Theorem 6, one can easily obtain

$$
\begin{gathered}
V(k+1)-\gamma V(k)+y^{T}(k) y(k)-\kappa^{2} w^{T}(k) w(k) \\
\leq \eta_{1}^{T}(k)\left[\begin{array}{ccccc}
\phi_{11} & \phi_{12} & \phi_{13} & 0 & \widetilde{\phi}_{15} \\
* & \phi_{22} & \phi_{23} & \phi_{24} & \widetilde{\phi}_{25} \\
* & * & \phi_{33} & 0 & 0 \\
* & * & * & \phi_{44} & 0 \\
* & * & * & * & \widetilde{\phi}_{55}
\end{array}\right] \eta_{1}(k) \\
+y^{T}(k) y(k)+z^{T}(k) W z(k)
\end{gathered}
$$

where

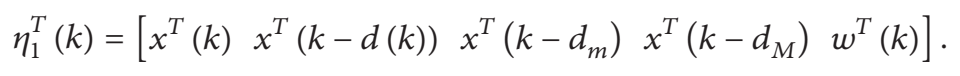

Combining (37) and (15), by Schur's complement, we have

$$
V(k+1)-\gamma V(k)+y^{T}(k) y(k)-\kappa^{2} w^{T}(k) w(k) \leq 0 .
$$

By Definition 7, the system (13) with time-varying delay satisfying (4) is globally exponentially stable with convergence rate $\lambda=\sqrt{\gamma}$ and $H_{\infty}$ performance $\kappa$ by the switching signal designed by (12).

Now, we extend Theorems 6 and 8 to obtain the corresponding results for uncertain switched systems (1). Set

$$
p(k, i)=\Delta_{i}(k)\left(N_{1 i} x(k)+N_{2 i} x(k-d(k))+N_{3 i} w(k)\right) .
$$

Combining (3), we have

$$
\begin{aligned}
p^{T}(k) p(k) \leq & \left(N_{1 i} x(k)+N_{2 i} x(k-d(k))+N_{3 i} w(k)\right)^{T} \\
& \times\left(N_{1 i} x(k)+N_{2 i} x(k-d(k))+N_{3 i} w(k)\right) .
\end{aligned}
$$

Then, for any $\varepsilon>0$, the following inequalities hold:

$$
\begin{aligned}
& \varepsilon\left(N_{1 i} x(k)+N_{2 i} x(k-d(k))+N_{3 i} w(k)\right)^{T} \\
& \quad \times\left(\left(N_{1 i} x(k)+N_{2 i} x(k-d(k))+N_{3 i} w(k)\right)\right. \\
& \quad-\varepsilon p^{T}(k, i) p(k, i) \geq 0 .
\end{aligned}
$$

By (2), the system (1) using (42) can be expressed as follows:

$$
\begin{aligned}
x(k+1)= & A_{i} x(k)+A_{d i} x(k-d(k)) \\
& +B_{i} w(k)+M_{1 i} p(k, i) \\
y(k)= & C_{i} x(k)+C_{d i} x(k-d(k)) \\
& +D_{i} w(k)+M_{2 i} p(k, i) .
\end{aligned}
$$

The following theorem provides the robust exponential stability conditions for uncertain switched systems (45) with $w(k)=0$.

Theorem 9. For some constants $\gamma \in(0,1]$ and $0 \leq \alpha_{i} \leq 1$, $i \in \mho, \sum_{i=1}^{N} \alpha_{i}=1$, if there exist positive definite symmetric matrices $P, U, Q_{j}, R_{j}(j=1,2,3)$ such that (15) and the following LMIs hold:

$$
\left[\begin{array}{cccccc}
\widetilde{\phi}_{11} & \widetilde{\phi}_{12} & 0 & 0 & \widetilde{\phi}_{16} & \phi_{15} \\
* & \widetilde{\phi}_{22} & \phi_{23} & \phi_{24} & \widetilde{\phi}_{26} & \phi_{25} \\
* & * & \phi_{33} & 0 & 0 & 0 \\
* & * & * & \phi_{44} & 0 & 0 \\
* & * & * & * & \widetilde{\phi}_{66} & M_{1 i}^{T} W^{T} \\
* & * & * & * & * & -W
\end{array}\right]<0,
$$


then the system (45) with time-varying delay satisfying (4) and $w(k)=0$ is globally exponentially stable with convergence rate $\lambda=\sqrt{\gamma}$ by the switching signal designed by (12).

$$
\text { Here }
$$

$$
\begin{array}{cc}
\widetilde{\phi}_{11}=\phi_{11}+\varepsilon N_{1 i}^{T} N_{1 i}, & \widetilde{\phi}_{12}=\phi_{12}+\varepsilon N_{1 i}^{T} N_{2 i}, \\
\tilde{\phi}_{16}=A_{i}^{T} P M_{1 i}, & \widetilde{\phi}_{22}=\phi_{22}+\varepsilon N_{2 i}^{T} N_{2 i}, \\
\tilde{\phi}_{26}=A_{d i}^{T} P M_{1 i}, & \widetilde{\phi}_{66}=M_{1 i}^{T} P M_{1 i}-\varepsilon .
\end{array}
$$

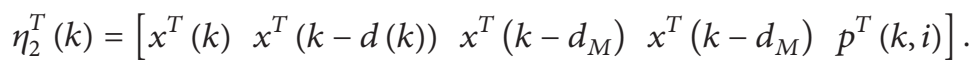

From (46) and (15), by Schur Complement, we have $V(k+1)-$ $\gamma V(k) \leq 0$.

Now, we are going to analyze the $H_{\infty}$ performance for the uncertain system (1). The main result is given as follows.

Theorem 10. For some constants $\gamma \in(0,1]$ and $0 \leq \alpha_{i} \leq 1$, $i \in \mho, \sum_{i=1}^{N} \alpha_{i}=1$, if there exist positive definite symmetric matrices $P, U, Q_{j}, R_{j}(j=1,2,3)$ such that (15) and the following LMIs hold

$$
\left[\begin{array}{cccccccc}
\widetilde{\phi}_{11} & \widetilde{\phi}_{12} & \phi_{13} & 0 & \bar{\phi}_{15} & \widetilde{\phi}_{16} & C_{i}^{T} & \phi_{15} \\
* & \widetilde{\phi}_{22} & \phi_{23} & \phi_{24} & \bar{\phi}_{25} & \widetilde{\phi}_{26} & C_{d i}^{T} & \phi_{25} \\
* & * & \phi_{33} & 0 & 0 & 0 & 0 & 0 \\
* & * & * & \phi_{44} & 0 & 0 & 0 & 0 \\
* & * & * & * & \bar{\phi}_{55} & \widetilde{\phi}_{56} & D_{i}^{T} & \widetilde{\phi}_{58} \\
* & * & * & * & * & \widetilde{\phi}_{66} & M_{2 i}^{T} & \widetilde{\phi}_{68} \\
* & * & * & * & * & * & -I & 0 \\
* & * & * & * & * & * & * & -W
\end{array}\right]<0
$$

$$
\eta_{3}^{T}(k)=\left[\begin{array}{lllll}
x^{T}(k) & x^{T}(k-d(k)) & x^{T}\left(k-d_{m}\right) & x^{T}\left(k-d_{M}\right) & w^{T}(k)
\end{array} p^{T}(k, i)\right] .
$$

then the system (1) with time-varying delay satisfying (4) is globally exponentially stable with convergence rate $\lambda=\sqrt{\gamma}$ and $H_{\infty}$ performance $\kappa$ by the switching signal designed by (12).

Here

$$
\begin{gathered}
\bar{\phi}_{15}=\tilde{\phi}_{15}+\varepsilon N_{1 i}^{T} N_{3 i}, \quad \bar{\phi}_{25}=\tilde{\phi}_{25}+\varepsilon N_{2 i}^{T} N_{3 i}, \\
\widetilde{\phi}_{58}=B_{i}^{T} W^{T}, \quad \bar{\phi}_{55}=\widetilde{\phi}_{55}+\varepsilon N_{3 i}^{T} N_{3 i}, \\
\widetilde{\phi}_{56}=B_{i}^{T} P M_{1 i}, \quad \widetilde{\phi}_{68}=M_{1 i}^{T} W^{T} .
\end{gathered}
$$

Proof. The result is carried out using the techniques employed for proving Theorems 6,8 , and 9 . There exists matrix $\Theta$ satisfying

$$
\begin{aligned}
& V(k+1)-\gamma V(k)+y^{T}(k) y(k)-\kappa^{2} w^{T}(k) w(k) \\
& \quad \leq \eta_{3}^{T}(k) \Theta \eta_{3}^{T}(k)+z^{T}(k) W z(k)+y^{T}(k) y(k),
\end{aligned}
$$

where
Combining (50) and (15), by Schur Complement, we have $V(k+1)-\gamma V(k)+y^{T}(k) y(k)-\kappa^{2} w^{T}(k) w(k) \leq 0$. 


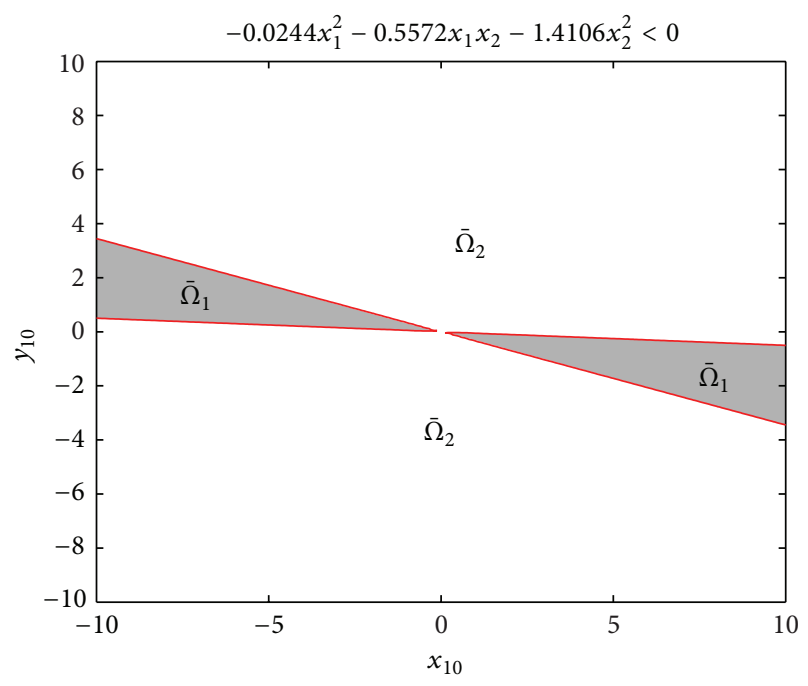

FIGURE 1: The switching regions.

TABLE 1: Allowable delay upper bound for different decay rate with $d_{m}=1, \alpha_{1}=\alpha_{2}=0.5$.

\begin{tabular}{lcc}
\hline & $\lambda=0.8$ & $\lambda=1$ \\
\hline$[26]$ & - & $d_{M}=2$ \\
{$[27]$} & $d_{M}=8$ & $d_{M}=182$ \\
Theorem 6 & $d_{M}=10$ & $d_{M}=\infty$ \\
\hline
\end{tabular}

Assume the minimum delay bound $d_{m}=1$. By Theorem 6, via solving LMIs (14) and (15) with $\lambda=0.8$ and $\alpha_{1}=\alpha_{2}=0.5$, we have $d_{M}=10$ and

$$
P=\left[\begin{array}{cc}
13.2019 & 30.1428 \\
* & 72.992
\end{array}\right], \quad U=\left[\begin{array}{cc}
0.4493 & 1.1242 \\
* & 3.098
\end{array}\right]
$$

From (9) and (11), one can obtain the switching regions

$$
\begin{gathered}
\bar{\Omega}_{1}=\left\{\left[\begin{array}{ll}
x_{1} & x_{2}
\end{array}\right]^{T} \in R:-0.0244 x_{1}^{2}-0.5572 x_{1} x_{2}\right. \\
\left.-1.4106 x_{2}^{2}<0\right\}, \quad \bar{\Omega}_{2}=R^{2} \backslash \bar{\Omega}_{1} .
\end{gathered}
$$

The switching regions $\bar{\Omega}_{1}$ and $\bar{\Omega}_{2}$ of the system are shown in Figure 1. Select the switching signal by

$$
\sigma(x(k))=i, \quad x(k) \in \bar{\Omega}_{i}, \quad i=1,2 .
$$

With the initial condition $\phi(\theta)=[1-1]^{T}, \theta=-10,-9, \ldots, 0$, and $d(k)=10$, the state responses of system are shown in Figure 2.

In order to show the improvement of our results over other recent results, some comparisons are made in Table 1. From Table 1, one can see that the results of this paper provide a larger allowable upper bound for time delay to guarantee the asymptotical or exponentially stability of system (1) with (54) by switching signal in (12).

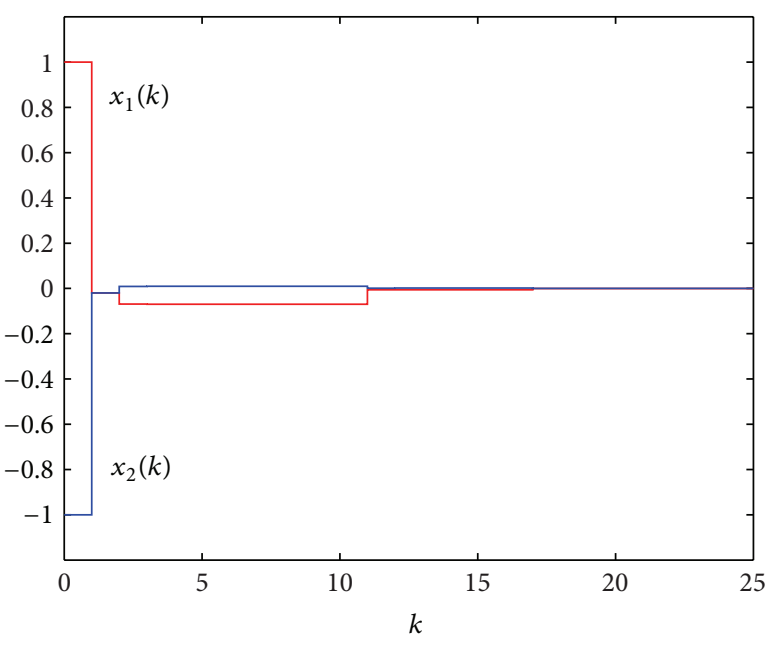

Figure 2: The state response.

Example 2 (see [27]). Consider system (13) with $w(k)=0$ and the following parameters:

$$
\begin{array}{ll}
A_{1}=\left[\begin{array}{cc}
1.01 & 0.1 \\
0 & 0.1
\end{array}\right], & A_{d 1}=\left[\begin{array}{cc}
-0.1 & 0 \\
-0.1 & -0.1
\end{array}\right], \\
A_{2}=\left[\begin{array}{cc}
0.1 & 0 \\
0.1 & 1.02
\end{array}\right], & A_{d 2}=\left[\begin{array}{cc}
0.12 & 0 \\
0.11 & 0.11
\end{array}\right] .
\end{array}
$$

Let $\alpha_{1}=\alpha_{2}=0.5$, the minimum delay bound $d_{m}=1$, and decay rate $\lambda=1$. The delay upper bound obtained by [27] is 3 . However, our result obtained by the application of Theorem 6 could be infinite to guarantee the asymptotic stability of system (13) with (58) by switching signal in (12). The switching regions can be designed in the same way as in Example 1.

Example 3. Consider the discrete-time switched system (13) with $w(k)=0$ and the following parameters:

$$
\begin{array}{ll}
A_{1}=\left[\begin{array}{cc}
1.21 & 0.1 \\
0 & 0.1
\end{array}\right], & A_{d 1}=\left[\begin{array}{cc}
-0.1 & 0 \\
-0.1 & -0.1
\end{array}\right], \\
A_{2}=\left[\begin{array}{cc}
0.1 & 0 \\
0.1 & 1.02
\end{array}\right], & A_{d 2}=\left[\begin{array}{cc}
0.12 & 0 \\
0.11 & 0.11
\end{array}\right] .
\end{array}
$$

One can easily find that both the above subsystems are unstable. The state responses of the two subsystems are shown in Figures 3 and 4 . Let $d_{m}=1$. By Theorem 6, via solving LMIs (14) and (15) with $\lambda=1$ and $\alpha_{1}=\alpha_{2}=0.5$, we have $d_{M}=5$ and

$$
\begin{aligned}
& P=1.0 e+003 *\left[\begin{array}{cc}
3.5486 & 0.1556 \\
* & 0.5634
\end{array}\right], \\
& U=1.0 e+003 *\left[\begin{array}{cc}
2.6204 & 0.2602 \\
* & 0.3184
\end{array}\right] .
\end{aligned}
$$




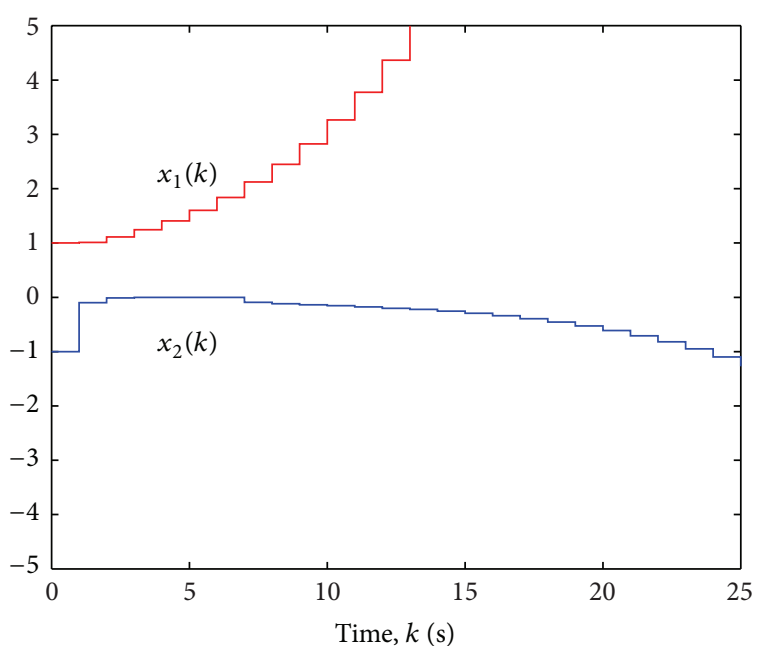

FIgURE 3: State responses of the subsystems 1.

From (9) and (11), one can obtain the switching regions

$$
\begin{gathered}
\bar{\Omega}_{i}^{1}=\left\{\left[\begin{array}{ll}
x_{1} & x_{2}
\end{array}\right]^{T} \in R: 2.5751 x_{1}^{2}+0.376 x_{1} x_{2}\right. \\
\left.-0.2741 x_{2}^{2}<0\right\}, \quad \bar{\Omega}_{2}=R^{2} \backslash \bar{\Omega}_{1} .
\end{gathered}
$$

Select the switching signal by

$$
\sigma(x(k))=i, \quad x(k) \in \bar{\Omega}_{i}, i=1,2
$$

With time-varying delay $d(k)=5$ and the initial condition $\phi(\theta)=\left[\begin{array}{ll}1 & -1\end{array}\right]^{T}, \theta=-5,-4, \ldots, 0$. The state responses of system are shown in Figure 5. It can be seen from Figure 5 that the designed switching law is effective although all subsystems are unstable. However, with the same parameters and $d_{M}=5$, the results in [27] cannot find any feasible solution to guarantee the asymptotic stability of system (13) with (59).

Example 4. Consider system (45) with the following parameters:

$$
\begin{array}{cc}
A_{1}=\left[\begin{array}{cc}
1 & 0.01 \\
0 & 0
\end{array}\right], & A_{d 1}=\left[\begin{array}{cc}
0 & 0.1 \\
0 & -0.1
\end{array}\right], \\
B_{1}=\left[\begin{array}{cc}
0.1 & 0 \\
0 & 0.1
\end{array}\right], & C_{1}=\left[\begin{array}{cc}
0.01 & 0 \\
0 & 0.08
\end{array}\right], \\
C_{d 1}=\left[\begin{array}{cc}
0.02 & 0 \\
0 & 0.01
\end{array}\right], & D_{1}=\left[\begin{array}{cc}
0.1 & 0.1 \\
0 & 0.2
\end{array}\right], \\
A_{2}=\left[\begin{array}{cc}
1 & 0.01 \\
0.01 & 1.01
\end{array}\right], & A_{d 2}=\left[\begin{array}{cc}
0.1 & 0 \\
0.1 & 0.1
\end{array}\right],
\end{array}
$$

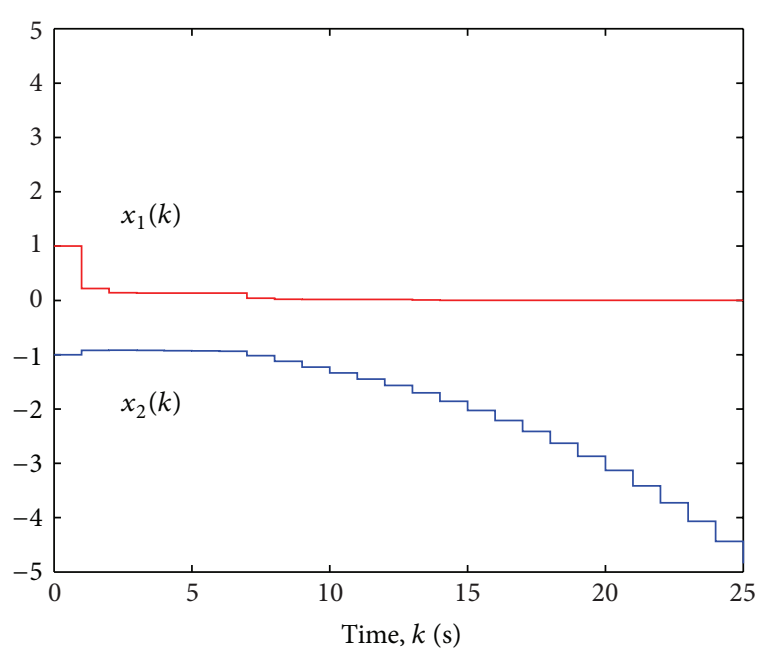

FIGURE 4: State responses of the subsystems 2.

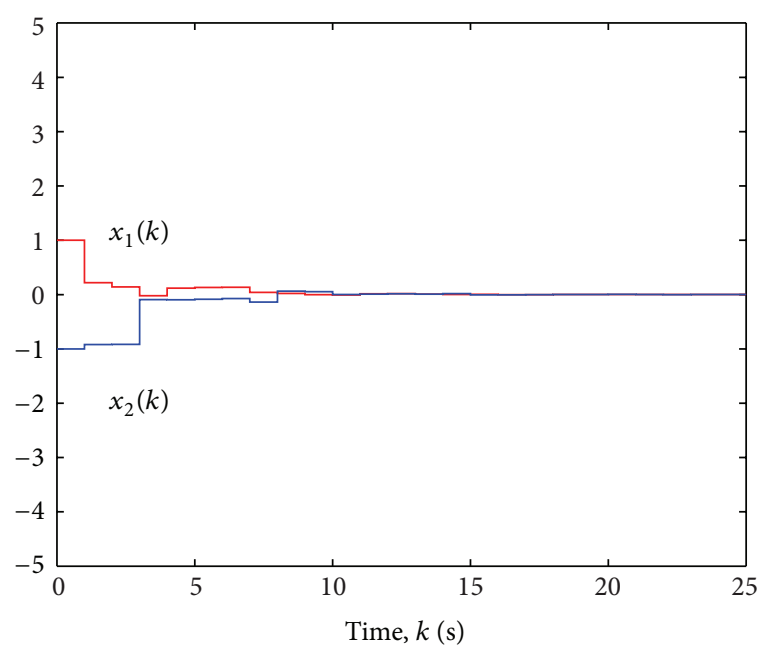

FIGURE 5: State responses of the switched systems 2.

$$
\begin{gathered}
B_{2}=\left[\begin{array}{cc}
0.2 & 0 \\
0.1 & 0.1
\end{array}\right], \quad C_{2}=\left[\begin{array}{cc}
0.03 & 0 \\
0 & 0.05
\end{array}\right], \\
C_{d 2}=\left[\begin{array}{cc}
0.02 & 0 \\
0 & 0.02
\end{array}\right], \quad D_{2}=\left[\begin{array}{cc}
0.2 & 0 \\
0 & 0.1
\end{array}\right], \\
M_{2}=\left[\begin{array}{cc}
0.1 & 0 \\
0 & 0.05
\end{array}\right], N_{2}=\left[\begin{array}{cc}
0.01 & 0 \\
0 & 0.02
\end{array}\right], \\
M_{1}=B_{1}, \quad N_{1}=C_{d 1}, \quad N_{3}=0.1 \times B_{1} .
\end{gathered}
$$

For a given $d_{m}=1, \gamma=0.98, \kappa=1.53$, and $\alpha_{1}=\alpha_{2}=0.5$. We compute the upper delay bound $d_{M}=23$ such that the uncertain system is exponential stability by applying Theorem 10 . However, with the same parameters and $d_{M}=23$, the results in [30] cannot find any feasible solution to guarantee 
the exponentially stable with $H_{\infty}$ performance $\kappa=1.53$ of system (45). Via solving LMIs (50) and (15), we have

$$
P=\left[\begin{array}{cc}
0.6836 & 0.0942 \\
* & 0.6615
\end{array}\right], \quad U=\left[\begin{array}{ll}
0.3424 & 0.0067 \\
0.0067 & 0.3387
\end{array}\right] \text {. }
$$

From (9) and (11), one can obtain the switching regions

$$
\begin{gathered}
\bar{\Omega}_{1}=\left\{\left[\begin{array}{ll}
x_{1} & x_{2}
\end{array}\right]^{T} \in R: 0.3413 x_{1}^{2}+0.0002 x_{1} x_{2}\right. \\
\left.-0.3386 x_{2}^{2}<0\right\}, \quad \bar{\Omega}_{2}=R^{2} \backslash \bar{\Omega}_{1} .
\end{gathered}
$$

Select the switching signal by $\sigma(x(k))=i, x(k) \in \bar{\Omega}_{i}, i=1,2$. On the other side, by setting the upper delay bound $d_{M}=3$, we have the $H_{\infty}$ performance $\kappa=0.275$.

\section{Conclusions}

By using improved discrete LK function combined with LMIs technique, in this paper, we propose new criteria for the exponential stability with $H_{\infty}$ performance of uncertain switched linear discrete-time systems with interval time-varying delay. If there is a feasible solution for the proposed LMIs conditions under some given upper bounds of delay, the switching law can be designed and the exponential stability of the systems can be achieved. Finally, the obtained results are shown to be less conservative than previous ones via the numerical examples.

\section{Acknowledgment}

This work was supported by the National Natural Science Foundation of China (nos. 61104064 and 61174038).

\section{References}

[1] Z. D. Sun and S. S. Ge, Switched Linear Systems-Control and Design, Springer, New York, NY, USA, 2004.

[2] D. Liberzon, "Basic problems in stability and design of switched systems," IEEE Control Systems Magazine, vol. 19, no. 5, pp. 5970, 1999.

[3] D. Liberzon, Switching in Systems and Control, Birkhäuser, Boston, Mass, USA, 2003.

[4] A. V. Savkin and R. J. Evans, Hybrid Dynamical Systems: Controller and Sensor Switching Problems, Springer, New York, NY, USA, 2002.

[5] M. Wicks, P. Peleties, and R. deCarlo, "Switched controller synthesis for the quadratic stabilization of a pair of unstable linear systems," European Journal of Control, vol. 4, no. 2, pp. 140-147, 1998.

[6] H. Lin and P. J. Antsaklis, "Stability and stabilizability of switched linear systems: a survey of recent results," IEEE Transactions on Automatic Control, vol. 54, no. 2, pp. 308-322, 2009.

[7] A. S. Morse, "Supervisory control of families of linear setpoint controllers-part 1: exact matching," IEEE Transactions on Automatic Control, vol. 41, no. 10, pp. 1413-1431, 1996.

[8] W. H. Chen and W. X. Zheng, "Delay-independent minimum dwell time for exponential stability of uncertain switched delay systems," IEEE Transactions on Automatic Control, vol. 55, no. 10, pp. 2406-2413, 2010.

[9] L. Zhang and P. Shi, "Stability $L_{2}$-gain and asynchronous $H_{\infty}$ control of discrete-time switched systems with average dwell time," IEEE Transactions on Automatic Control, vol. 54, no. 9, pp. 2192-2199, 2009.

[10] G. Zhai, B. Hu, K. Yasuda, and A. N. Michel, "Stability analysis of switched systems with stable and unstable subsystems: an average dwell time approach," in Proceedings of the American Control Conference, pp. 200-204, 2000.

[11] X. D. Zhao, L. X. Zhang, P. Shi, and M. Liu, "Stability and stabilization of switched linear systems with mode-dependent average dwell time," IEEE Transactions on Automatic Control, vol. 57, no. 7, pp. 1809-1815, 2012.

[12] X. D. Zhao, H. Liu, and Z. H. Wang, "Weighted $H_{\infty}$ performance analysis of switched linear systems with modedependent average dwell time," International Journal of System Science, vol. 44, no. 11, pp. 2130-2139, 2013.

[13] M. Johansson and A. Rantzer, "Computation of piecewise quadratic Lyapunov functions for hybrid systems," IEEE Transactions on Automatic Control, vol. 43, no. 4, pp. 555-559, 1998.

[14] A. Bacciotti, "Stabilization by means of state space depending switching rules," Systems \& Control Letters, vol. 53, no. 3-4, pp. 195-201, 2004.

[15] J. K. Hale and S. M. Verduyn Lunel, Introduction to FunctionalDifferential Equations, Springer, New York, NY, USA, 1993.

[16] J. N. Li, H. Y. Su, Z. Z. Wu, and J. Chu, "Robust stabilization for discrete-time nonlinear singular systems with mixed time delays," Asian Journal of Control, vol. 15, no. 6, pp. 1-11, 2013.

[17] Z. G. Feng, J. Lam, and H. J. Gao, "Delay-dependent robust $H_{\infty}$ controller synthesis for discrete singular delay systems," International Journal of Robust and Nonlinear Control, vol. 21, no. 16, pp. 1880-1902, 2011.

[18] Q.-L. Han, "A discrete delay decomposition approach to stability of linear retarded and neutral systems," Automatica, vol. 45, no. 2, pp. 517-524, 2009.

[19] Y. G. Sun, L. Wang, and G. Xie, "Delay-dependent robust stability and stabilization for discrete-time switched systems with mode-dependent time-varying delays," Applied Mathematics and Computation, vol. 180, no. 2, pp. 428-435, 2006.

[20] W. A. Zhang and L. Yu, "Stability analysis for discrete-time switched time-delay systems," Automatica, vol. 45, no. 10, pp. 2265-2271, 2009.

[21] C. Meyer, S. Schroder, and R. W. de Doncker, "Solid-state circuit breakers and current limiters for medium-voltage systems having distributed power systems," IEEE Transactions on Power Electronics, vol. 19, no. 5, pp. 1333-1340, 2004.

[22] P. Yan and H. Ozbay, "Stability analysis of switched time delay systems," SIAM Journal on Control and Optimization, vol. 47, no. 2, pp. 936-949, 2008.

[23] S. Kim, S. A. Campbell, and X. Z. Liu, "Stability of a class of linear switching systems with time delay," IEEE Transactions on Circuits and Systems, vol. 53, no. 2, pp. 384-393, 2006.

[24] X. M. Sun, W. Wang, G. P. Liu, and J. Zhao, "Stability analysis for linear switched systems with time varying delay," IEEE Transactions on Systems, Man and Cybernetics B, vol. 38, pp. 528-533, 2008.

[25] V. N. Phat, "Robust stability and stabilizability of uncertain linear hybrid systems with state delays," IEEE Transactions on Circuits Systems II-Express Briefs, vol. 52, no. 2, pp. 94-98, 2005. 
[26] V. N. Phat and K. Ratchagit, "Stability and stabilization of switched linear discrete-time systems with interval timevarying delay," Nonlinear Analysis: Hybrid Systems, vol. 5, no. 4, pp. 605-612, 2011.

[27] C. H. Lien, K. W. Yu, H. C. Chang, L. Y. Chung, and J. D. Chen, "Switching signal design for exponential stability of discrete switched systems with interval time-varying delay," Journal of the Franklin Institute, vol. 349, no. 6, pp. 2182-2192, 2012.

[28] C. H. Lien, K. W. Yu, Y. J. Chung, H. C. Chang, and J. D. Chen, "Switching signal design for global exponential stability of uncertain switched nonlinear systems with time-varying delay," Nonlinear Analysis: Hybrid Systems, vol. 5, no. 1, pp. 10-19, 2011.

[29] Y. G. Sun, L. Wang, and G. Xie, "Delay-dependent robust stability and control for uncertain discrete-time switched systems with mode-dependent time delays," Applied Mathematics and Computation, vol. 187, no. 2, pp. 1228-1237, 2007.

[30] C. H. Lien, K. W. Yu, L. Y. Chung, and J. D. Chen, " $H_{\infty}$ performance for uncertain discrete switched systems with interval time-varying delay via switching signal design," Applied Mathematical Modelling, vol. 37, no. 4, pp. 2484-2494, 2013.

[31] F. Uhlig, "A recurring theorem about pairs of quadratic forms and extensions," Linear Algebra and Its Applications, vol. 25, pp. 219-237, 1979.

[32] S. P. Boyd, El Ghaoui, E. Feron, and V. Balakrishnan, Linear Matrix Inequalities in System and Control Theory, SIAM, Philadelphia, Pa, USA, 1994. 


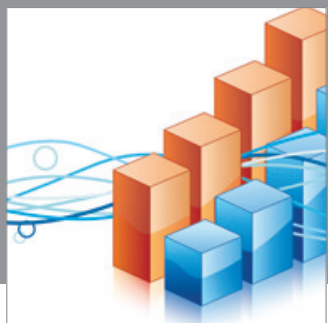

Advances in

Operations Research

mansans

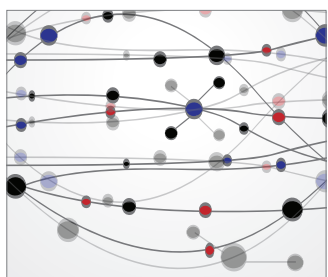

The Scientific World Journal
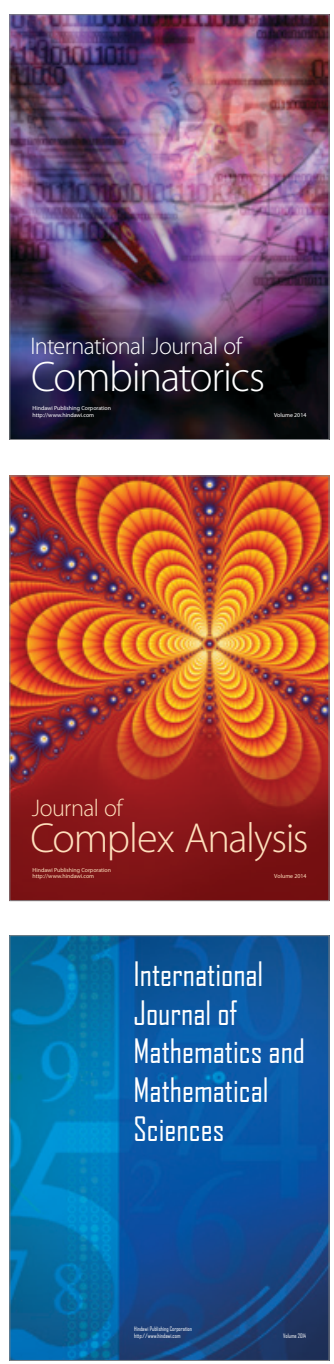
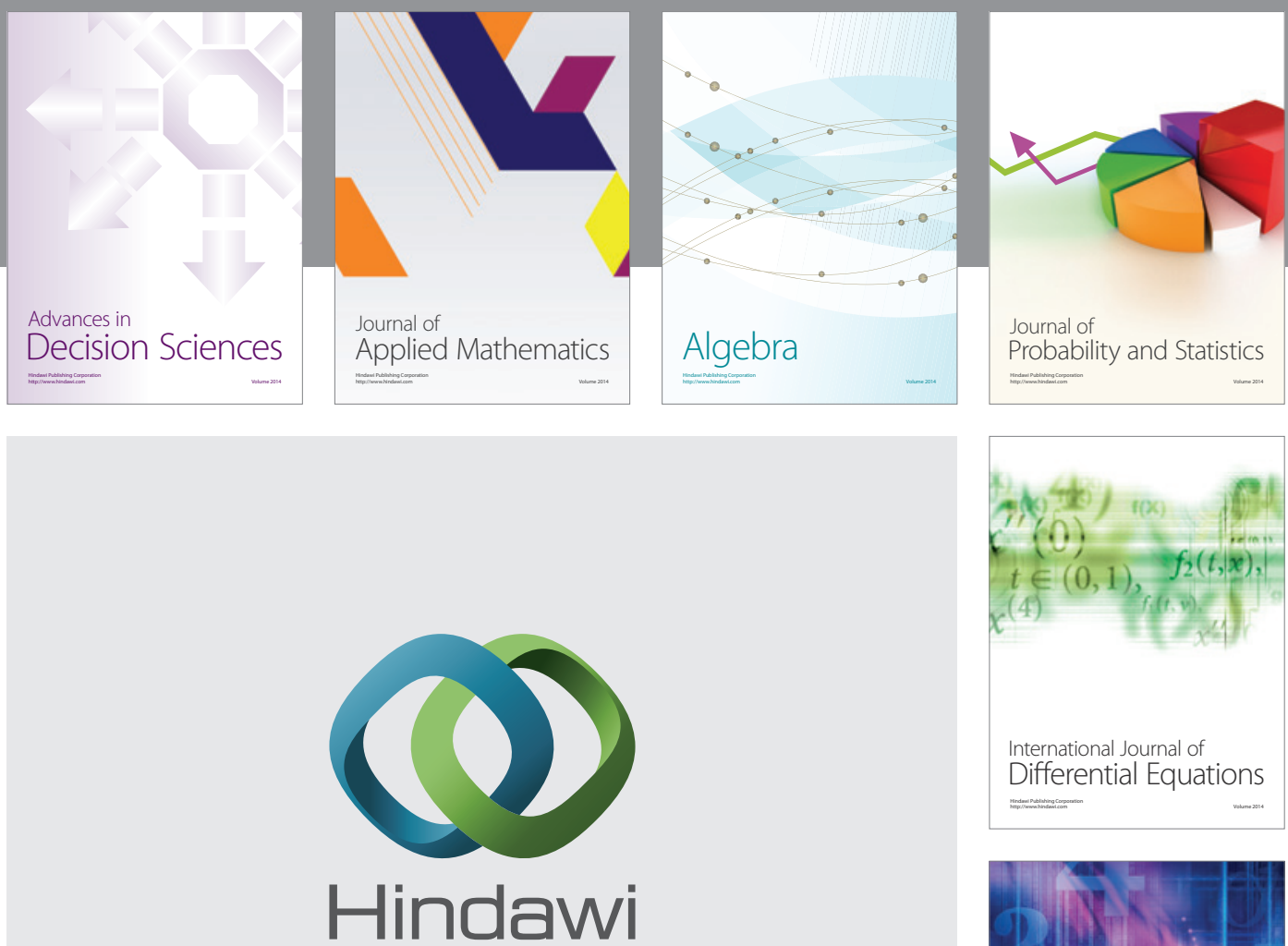

Submit your manuscripts at http://www.hindawi.com
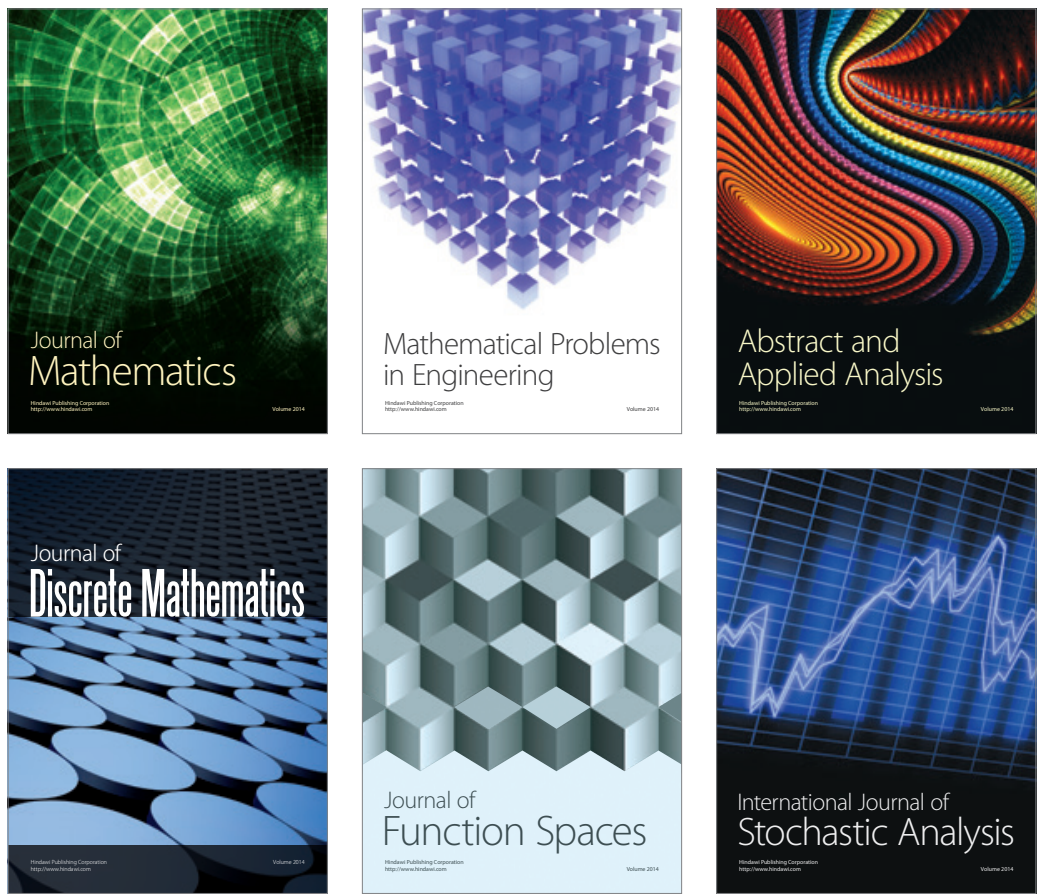

Journal of

Function Spaces

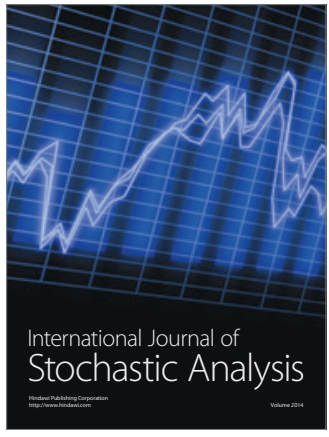

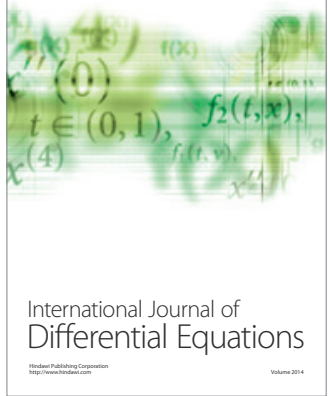
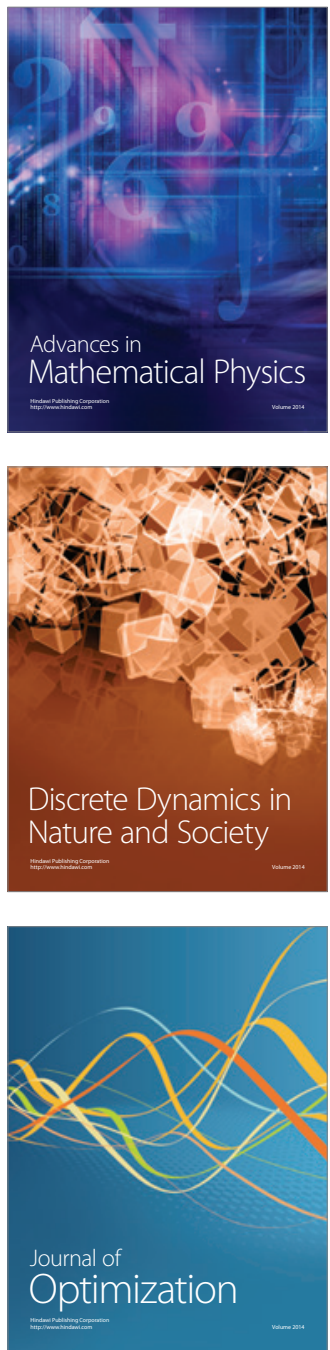